\title{
Data-Driven Abnormal Condition Identification and Self-Healing Control System for Fused Magnesium Furnace
}

\author{
Zhiwei Wu, Yongjian Wu, Tianyou Chai, Fellow, IEEE, and Jing Sun, Fellow, IEEE
}

\begin{abstract}
In the smelting process of fused magnesium furnaces (FMFs), frequent changes in the raw material granule size and impurity constituent will cause the arc resistance between the lower end of the electrode and the surface of the molten pool to vary, and thus, the smelting currents fluctuate. Consequently, abnormal conditions, which can arise if the setpoints of electrode currents are not properly adjusted on time, will causethe performance to deteriorate or even the overall operation to stall. Through analysis of the characteristics of different operating conditions, this paper presents a data-driven abnormal condition identification and self-healing control system. The proposed system extracts the identification rules according to the current tracking error, as well as the rate and duration of the current fluctuations, and identifies the abnormal conditions based on rule-based reasoning. The selfhealing control is developed using case-based reasoning to correct the current setpoints based on the identification results. The outputs of the control loop track the corrected setpoints, thereby forcing the process to recover from the abnormal conditions. The proposed method and the developed control system have been applied to a real FMF, and substantial improvement is achieved with many benefits provided to the factory. The implementation results show that occurrence of abnormal conditions has been reduced by more than $50 \%$, and the product quality has been increased by more than $2 \%$.
\end{abstract}

Index Terms-Abnormal conditions identification, casebased reasoning (CBR), data-driven, fused magnesium furnace (FMF), rule-based reasoning (RBR), self-healing control.

\section{INTRODUCTION}

$\mathbf{F}$ USED magnesia with features such as a high melting point, robust structural integrity, strong insulating proper-

Manuscript received May 4, 2014; revised May 18, 2014 and June 19, 2014; accepted June 24, 2014. Date of publication August 19, 2014; date of current version February 6, 2015. This work was supported in part by the Natural Science Foundation of China under Grant 61020106003, Grant 61203102, and Grant 61333012, and in part by the 111 Project of the Ministry of Education of China under Grant B08015. (Corresponding author: Yongjian Wu.)

Z. Wu, Y. Wu, and T. Chai are with the State Key Laboratory of Synthetical Automation for Process Industries, Northeastern University, Shenyang 110819, China, and also with the Research Center of Automation, Northeastern University, Shenyang 110819, China (e-mail: wuzhiwei_2006@163.com; wyj_neu@163.com; tychai@mail. neu.edu.cn).

$\mathrm{J}$. Sun is with the Naval Architecture and Marine Engineering Department, University of Michigan, Ann Arbor, MI 48109 USA (e-mail: jingsun@umich.edu).

Color versions of one or more of the figures in this paper are available online at http://ieeexplore.ieee.org.

Digital Object Identifier 10.1109/TIE.2014.2349479 ties, and good antioxidation is an important refractory for many industries such as metallurgical, chemical, electric apparatus, and aerospace industry. China has a rich magnesite reserve, counting up to approximately a quarter of the world. Due to the special characteristics of China's magnesite material, such as low-grade, complex mineral composition, and large variations in the composition, high-purity fused magnesia is produced mainly by the unique three-phase ac fused magnesium furnace (FMF). The FMF adjusts the distances between the three-phase electrodes and the surface of molten pool appropriately to control the current values of three-phase electrode and the intensity of the electrical arc. The raw magnesite materials, whose main ingredient is $\mathrm{MgCO}_{3}$, are melted by the heat released by arcs in the FMF and then coagulated into the final products. The power consumed is the largest cost item in the smelting process, it mounts typically to more than $20000 \mathrm{kWh}$ for a batch and accounts to more than $60 \%$ of the total cost. Therefore, the control objective of FMF is to control the product quality within a desired range, while minimizing the energy consumption per ton.

Smelting is a complex process that involves many different phases. The normal smelting process includes three operating conditions: heating and melting, feeding, and exhausting. The control of the operating condition is mainly achieved by controlling the currents through properly adjusting the gaps between the three-phase electrodes and the surface of molten pool. Therefore, controlling the currents in a stable and optimal manner in the normal smelting process is important to reduce the energy consumption per ton. Reference [1] introduces an electrode current switching control strategy based on simultaneous perturbation stochastic approximation (SPSA) and rulebased reasoning (RBR), which has been successfully used in the industrial applications. In the FMF smelting process, heat energy used for melting the raw materials is completely converted from electric energy. As the smelting voltage and time are constant, the electric energy consumption is directly related to the three-phase electrodes currents. Large current leads to increased energy consumption. Therefore, the energy consumption per ton product is dictated by the current setpoints. Under the premise that the melting point, resistivity and granule size of the raw materials are within the normal range, [2] and [3] present electrode current setting strategies for three normal operating conditions based on case-based reasoning (CBR) and RBR combined with proportional- integral compensation, respectively.

During the FMF smelting process, however, the raw material granule sizes and impurity constituents (mainly $\mathrm{SiO}_{2}, \mathrm{Al}_{2} \mathrm{O}_{3}$, 
$\mathrm{Fe}_{2} \mathrm{O}_{3}$ ) are frequently changed, and these changes will cause fluctuations in melting point and resistivity of the raw materials. If the current setpoints are not adjusted when the particle size, meting point, and resistivity of raw materials change, the following abnormal conditions will occur.

1) Semimolten condition: During the heating and smelting condition, the raw materials cannot be melted fully when the melting point increases. This condition will lead to rapid increase in the temperature at some local area of the furnace wall and even the eventual melting the furnace wall.

2) Overheating condition: When the melting point of raw material is decreased, the molten pool rises so fast that it exceeds the allowable range of the smelting process. This condition will lead to product quality degradation as the impurity content tends to increase.

3) Abnormal feeding condition: In the feeding operation, the currents decrease when the raw material resistivity increases, then the electrodes move down frequently, and the new low-temperature raw materials fall directly into the molten pool. This condition can lead to low product yield.

4) Abnormal exhausting condition: The particle size of the raw materials determines the gap between the raw materials and the electrodes. When the particle size exceeds the normal range, the pressure inside the FMF will not be balanced with the atmospheric pressure. In serious conditions, the high-temperature melts will spurt out of the furnace together with the gas, even stall the smelting process.

The abnormal conditions of the FMF smelting process are typically caused by the existing current control system, which does not properly adjust the current setpoints when the changes in melting point, resistivity and particle size of raw materials lead to the arc resistance fluctuate and cause the currents change. The arc resistance dynamical characteristics are influenced by the ingredients, the temperature distribution, the electrode consumption and the level of molten pool. These factors are difficult to measure online. In general, the relationship of the abnormal conditions and the arc resistance have strong nonlinearity, it is very difficult to obtain accurate models for abnormal conditions because the mechanisms causing the abnormal conditions are not fully understood. For complex industrial processes, the data-driven fault diagnosis methods have been applied extensively in recent years [4]-[11]. Reference [6] proposed a model-data integrated fault tolerant control scheme with performance optimization for real-time industrial applications. Reference [7] provides a comparison study on the data-driven multivariate statistical analysis methods, and illustrates the efficiencies of all the discussed methods for fault diagnosis according to the fault data of industrial benchmark of Tennessee Eastman process. The result of [7] serves as a reference for basic data-driven fault diagnosis and process monitoring schemes for modern complex processes. Reference [10] and [11], based on multivariable statistical analysis and subspace separation, used current data to achieve the fault diagnosis for FMF smelting process; however, these two papers cannot identify the fault types for FMF. The self-healing control strategy is applied to power systems [12]-[14] and industrial wireless sensor networks [15]. When a fault occurs, the network structure reconstruction methods are used to eliminate the fault, and for the reconstructed systems its robust stability can be done by [16]-[18]. To better track the desired economic objectives, [19] proposes a networked multirate output feedback control approach for setpoints compensation, which has been successfully used in a rougher flotation process. However, faults for the complex industrial process can arise if the setpoints of the control loops are improper. For the special faults of the shaft-furnace roasting process caused by improperly defined setpoints, [20] diagnoses the faults based on the rules extracted by expert experiences, and then the self-healing controller adjusts the setpoints of the control loop to drive the shaft-furnace roasting process gradually out of faulty operating conditions. The aforementioned fault diagnosis and self-healing methods are applied to diagnose and deal with the faults in actuators, sensors or specific industrial processes, but they are difficult to apply to the FMF smelting process.

This paper presents a data-driven abnormal condition identification and self-healing control strategy for the FMF. The proposed method extracts the rules according to the current tracking errors, the change rate of the currents and the duration for currents fluctuation, and identifies the abnormal conditions based on RBR, and then autoadjusts the setpoints of the control loop for the three-phase electrode currents using a CBR-based algorithm. The corrected setpoints are well tracked by the control loop so that the abnormal conditions can be eliminated. Industrial applications show that the proposed data-driven abnormal condition identification and self-healing control system are effective and yield substantial benefits in terms of cost saving and product quality improvement.

The organization of this paper is as follows. Section II discusses the control process and the abnormal conditions of FMF. In Section III, the abnormal condition identification and selfhealing control strategy are expounded. The performance of the proposed method in industrial field is analyzed in Section IV, followed by conclusions in Section V.

\section{Analysis of the ABnormal CONDitions of FMF SMELTing CONTROL Process}

\section{A. FMF Smelting Control Process}

The FMF smelting control process is shown in Fig. 1. It includes the power supply system consisting of transformer and transducer, the current control system consisting of current optimal setting and current controller, electrode position control system consisting of three motors and three electrodes.

At the beginning of the FMF smelting process, a small amount of raw materials is fed into the furnace from the storage silo at the top of FMF. According to the desired values of energy consumption per ton $r^{*}$ and range, the voltages $U$, the raw material granule size $B_{1}$ and raw material impurity constituent $B_{2}$, the current control system generates the current setpoints $\hat{y}_{1}(t)$ for heating and melting condition. According to the tracking error $e_{j}(t)$ between the current $y_{j}(t)(j=1, \ldots, 3)$ and $\hat{y}_{1}(t)$, the three-phase electrodes are moved up and down by 


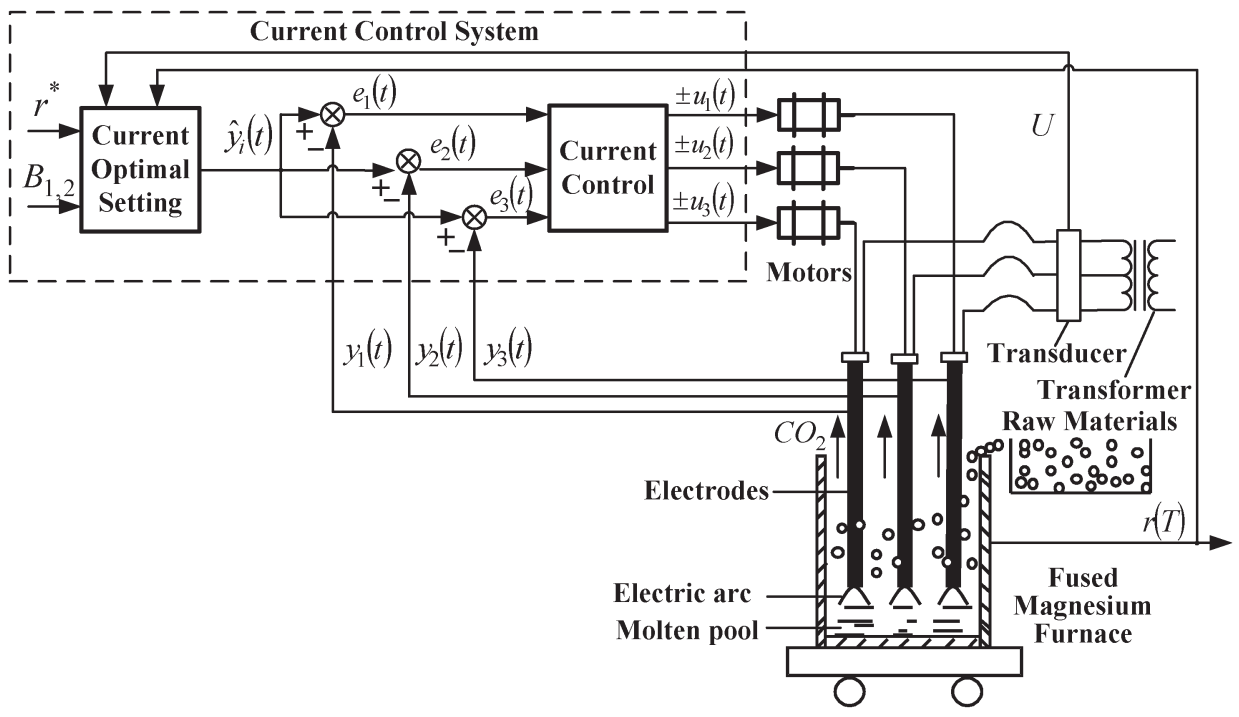

Fig. 1. Diagram of FMF smelting control process.

adjusting the rotate speed and direction of the motors $\pm u_{j}(t)$ to put the electrodes in suitable positions to form the electric arcs between the electrodes and the raw material. The raw materials are melted by absorbing heat released by the arcs and then the molten pool can be formed below the electrodes. With the raw materials melting, raw magnesites need to be fed into the furnace. According to the technical requirements, the currents need to be reduced in the feeding condition. The control system will adjust the current setpionts to $\hat{y}_{2}(t)\left(\hat{y}_{2}(t)<\hat{y}_{1}(t)\right)$, and control the currents $y_{j}(t)$ to track $\hat{y}_{2}(t)$. The chemical reactions in FMF will produce a certain amount of $\mathrm{CO}_{2}$ gas during the smelting process, and $\mathrm{CO}_{2}$ gas has to be displaced. Under the exhausting condition, the current setpoints are adjusted to $\hat{y}_{3}(t)\left(\hat{y}_{3}(t)<\hat{y}_{1}(t)\right)$ and the control system will track the new setpoints to increase the motion of the electrodes to facilitate the gas exhaustion. A normal smelting process will go through the aforementioned three conditions periodically. Heating and melting is the operating condition for most of the time. As the smelting process being carried on, it will also assume the feeding and exhausting conditions from time to time.

\section{B. Abnormal Condition Characteristic Analysis}

The heating and melting, feeding, and exhausting conditions will be expressed as $S_{a}, S_{b}$, and $S_{c}$, respectively, in the sequel. During the aforementioned three conditions, improper current setpoints will result in four abnormal conditions and their corresponding characteristics are analyzed as follows:

1) The heating and meting condition $S_{a}$ is the normal smelting process of raw materials. The influence factors for $S_{a}$ include the smelting voltage $U$, the current setpoint $\hat{y}_{1}(t)$ for $S_{a}$, the three-phase electrode currents $y_{j}(t)$ and the arc resistance $R_{\text {arc }}^{a}$ for $S_{a}$, as shown in

$$
S_{a}(t)=F_{a}\left(U, \hat{y}_{1}(t), y_{1}(t), y_{2}(t), y_{3}(t), R_{\mathrm{arc}}^{a}\right)
$$

where $F_{a}(\cdot)$ is an unknown nonlinear function to describe the relationship between the influence factors and the $S_{a}$. In addition, the arc resistance $R_{\mathrm{arc}}^{a}$ is related to the control system output vectors $u_{j}(u)$, the melting point of raw materials $T_{\mathrm{mp}}$, the resistivity of raw materials $\rho_{\mathrm{rm}}$, and the impurity constituent of raw materials $B_{2}$, this relationship can be described as follows:

$$
R_{\mathrm{arc}}^{a}=f_{\mathrm{arc}}^{a}\left(u_{1}(t), u_{2}(t), u_{3}(t), T_{\mathrm{mp}}, \rho_{\mathrm{rm}}, B_{2}\right)
$$

where $f_{\operatorname{arc}}^{a}(\cdot)$ is an unknown nonlinear functions.

During the heating and melting condition, when $\mathrm{MgCO}_{3}$ is heated from the room temperature to $640{ }^{\circ} \mathrm{C}-800{ }^{\circ} \mathrm{C}$, it decomposes into $\mathrm{CO}_{2}$ gas and solid state of $\mathrm{MgO} . \mathrm{MgO}$ continues to be heated up to $2800{ }^{\circ} \mathrm{C}$ when it turns into molten state, and the molten pool be formed below the electrodes. The physical and chemical changes in smelting process are shown as follows:

$$
\begin{aligned}
& \mathrm{MgCO}_{3} \stackrel{640{ }^{\circ} \mathrm{C}-800{ }^{\circ} \mathrm{C}}{\longrightarrow} \mathrm{CO}_{2}+\mathrm{MgO} \text { (solid state) } \\
& \mathrm{MgO} \text { (solid state) } \stackrel{2800{ }^{\circ} \mathrm{C}}{\longrightarrow} \mathrm{MgO} \text { (liquid state). }
\end{aligned}
$$

If the melting point of the raw materials does not change, the heat released by the arc will be in steady state and the temperature in the furnace can be maintained in a proper range $\left(2800{ }^{\circ} \mathrm{C}-2850^{\circ} \mathrm{C}\right)$ without great fluctuation, as long as the three-phase electrode currents track the setpoints properly. This corresponds to the normal heating and melting condition in the smelting process, and will be referred to as $S_{a}^{1}$. In $S_{a}^{1}$, the reaction of (4) is sufficient, and the melt rates of raw materials are appropriate, this condition tends to help improve the product quality and reduce the energy consumption per ton.

When the melting point of the raw materials $T_{\mathrm{mp}}$ is increased, caused by the changes in $B_{2}$, the temperature inside the FMF can go below the melting point if the current setpoints $\hat{y}_{1}(t)$ are not adjusted on time. Raw materials without being fully melted will stay in the semimolten state and fall into the molten pool, where the reactions of (3) and (4) will take place. This will cause the pool level to increase, reducing the arc lengths between the lower end of the electrode and surface of the 
molten pool and the arc resistance $R_{\mathrm{arc}}^{a}$, thereby leading to an increase in the currents $y_{j}(t)$ if the smelting voltage $U$ is constant. When the duration for this current fluctuation $T_{1}$ exceeds the time threshold, too much gas will be gathered in the molten pool, high-temperature melting liquids could penetrate the insulation layer around the furnace wall. This will lead to a rapid increase in the temperature at some local area of the furnace wall and even cause the eventual melting of the furnace wall. This corresponds to the semimelting condition, denoted as $S_{a}^{2}$.

When the melting point $T_{\mathrm{mp}}$ decreases, the constant current setpoints $\hat{y}_{1}(t)$ will result in the temperature inside the FMF being higher than the melting point. A large amount of raw materials are melted in a short time, and the molten pool rises so fast that it exceeds the allowable range of the smelting process. The rapid rise of the molten pool will cause the arc resistance $R_{\text {arc }}^{a}$ to decrease, the currents $y_{j}(t)$ increase and the impurity in the molten pool cannot be separated out thoroughly. When the duration for the current fluctuation $T_{1}$ exceeds the threshold, the overheating condition $S_{a}^{3}$ happens. Overheating condition will lead to energy waste and product quality degradation, as consequences of increased impurity content in this condition.

2) The nonlinear relationship between the feeding condition $S_{b}$ and its influence factors is as follows:

$$
S_{b}(t)=F_{b}\left(U, \hat{y}_{2}(t), y_{1}(t), y_{2}(t), y_{3}(t), R_{\mathrm{arc}}^{b}\right)
$$

where $F_{b}(\cdot)$ is an unknown nonlinear function, $R_{\text {arc }}^{b}$ denotes the arc resistance for feeding condition, which depends on $u_{j}(t), T_{\mathrm{mp}}, \rho_{\mathrm{rm}}$ and $B_{2}$ and is described as follows:

$$
R_{\mathrm{arc}}^{b}=f_{\mathrm{arc}}^{b}\left(u_{1}(t), u_{2}(t), u_{3}(t), T_{\mathrm{mp}}, \rho_{\mathrm{rm}}, B_{2}\right)
$$

where $f_{\mathrm{arc}}^{b}(\cdot)$ is an unknown nonlinear functions and different from $f_{\text {arc }}^{a}(\cdot)$.

According to the process requirements, the downward movements of electrodes should be avoided during normal feeding condition (expressed as $S_{b}^{1}$ ) to prevent the new low-temperature raw materials to slide into the molten pool directly and affect the product quality. The changes in $B_{2}$ will cause the resistivity of raw materials $\rho_{\text {rm }}$ to increase, and the currents $y_{j}(t)$ to reduce. In this case, if the current setpoints $\hat{y}_{2}(t)$ is not adjusted, the current control system has to frequently adjust the electrode position down to track the current setpoints. The raw materials ( solid $\mathrm{MgCO}_{3}$ ), without adequate heating, will slide into the molten pool directly along the axial surface of the electrodes, causing the fluctuation of the surface of the molten pool, consequently the arc resistance $R_{\text {arc }}^{b}$ becomes unstable and the currents $y_{j}(t)$ will fluctuate. If the duration for current fluctuation $T_{2}$ exceeds the threshold, the low temperature raw material slide into molten pool will affect the uniform distribution of the temperature field in the pool, and thus the product qualities will deteriorate. This will be referred to as the abnormal feeding condition $S_{b}^{2}$.
3) The exhausting condition $S_{c}$ is influenced by several factors

$$
S_{c}(t)=F_{c}\left(U, \hat{y}_{3}(t), y_{1}(t), y_{2}(t), y_{3}(t), R_{\mathrm{arc}}^{c}\right)
$$

where $F_{c}(\cdot)$ is an unknown nonlinear function, $R_{\text {arc }}^{c}$ denotes the arc resistance for exhausting condition, which can be expressed as

$$
R_{\mathrm{arc}}^{c}=f_{\mathrm{arc}}^{c}\left(u_{1}(t), u_{2}(t), u_{3}(t), T_{\mathrm{mp}}, \rho_{\mathrm{rm}}, B_{1}\right) .
$$

In normal exhausting conditions (expressed as $S_{c}^{1}$ ), if the raw material particle size $B_{1}$ is appropriate, the current control system tracks the setpoints $\hat{y}_{3}(t)$ to increase the movement of the electrodes, which can produce a gap between the electrodes and the raw materials to facilitate the $\mathrm{CO}_{2}$ gas exhaustion.

When the particle size $B_{1}$ changes (bigger or smaller), the gap between the electrodes and the raw materials will also change. If the current setpoints of exhausting condition $\hat{y}_{3}(t)$ is not adjusted, the changed gap will not be appropriate and the pressure inside the FMF will not be balanced with the atmospheric pressure. In this case, the fluctuations of the pool level and the position of the three-phase electrodes will be very large, causing large variations in the arc resistance $R_{\text {arc }}^{c}$ and in the currents $y_{j}(t)$. If the duration for current fluctuation $T_{3}$ is too long, the high-temperature melts will spurt out of the furnace together with the $\mathrm{CO}_{2}$ gas, leading to the abnormal exhausting condition $S_{c}^{2}$. As the temperature of the melt exceeds $2800{ }^{\circ} \mathrm{C}$, this condition will pose a great threat to the safety of the equipments and the operators.

\section{Manual Handling Reality for Abnormal Conditions}

The changes in raw material particle size $B_{1}$ or impurity constituent $B_{2}$ will cause the arc resistances $R_{\text {arc }}^{a, b, c}$ to change, which, in turn, will cause fluctuation of the currents $y_{j}(t)$. In such scenario, if the current setpoints $\hat{y}_{i}(t)$ are unchanged, the abnormal conditions will happen. While the qualitative connections between the abnormal conditions and $T_{\mathrm{mp}}, \rho_{\mathrm{rm}}$ and $B_{1}$ are established, the mechanisms are not fully understood and their mathematical models are difficult to be developed because $T_{\mathrm{mp}}$, $\rho_{\text {rm }}$ and $B_{1}$ are influenced by some unmeasurable variables such as impurity constituent of raw materials, temperature distribution in FMF, uniformity of the distribution of raw materials and so on. Moreover, $R_{\text {arc }}^{a, b, c}$ cannot be measured online. Currently, the abnormal conditions of FMF are primarily identified and dealt with manually based on operators' experience, as shown by Fig. 2. The operators observe the fluctuations of the three-phase electrode currents and look out for special phenomena of the smelting equipment (including some local "bright spot" on the outer furnace wall, unusual fast melting speed of raw materials, spurting out of high-temperature melts, etc.) to detect the abnormal conditions. When the abnormal conditions are detected, the operators determine the amount of adjustment $\Delta \hat{y}_{i}(t)(i=1, \ldots, 3)$ of the current setpoints for heating and melting, feeding, and exhausting conditions, and adjust the current setpoints $\bar{y}_{i}(t)=\hat{y}_{i}(t)+\Delta \hat{y}_{i}(t)$ accordingly. 


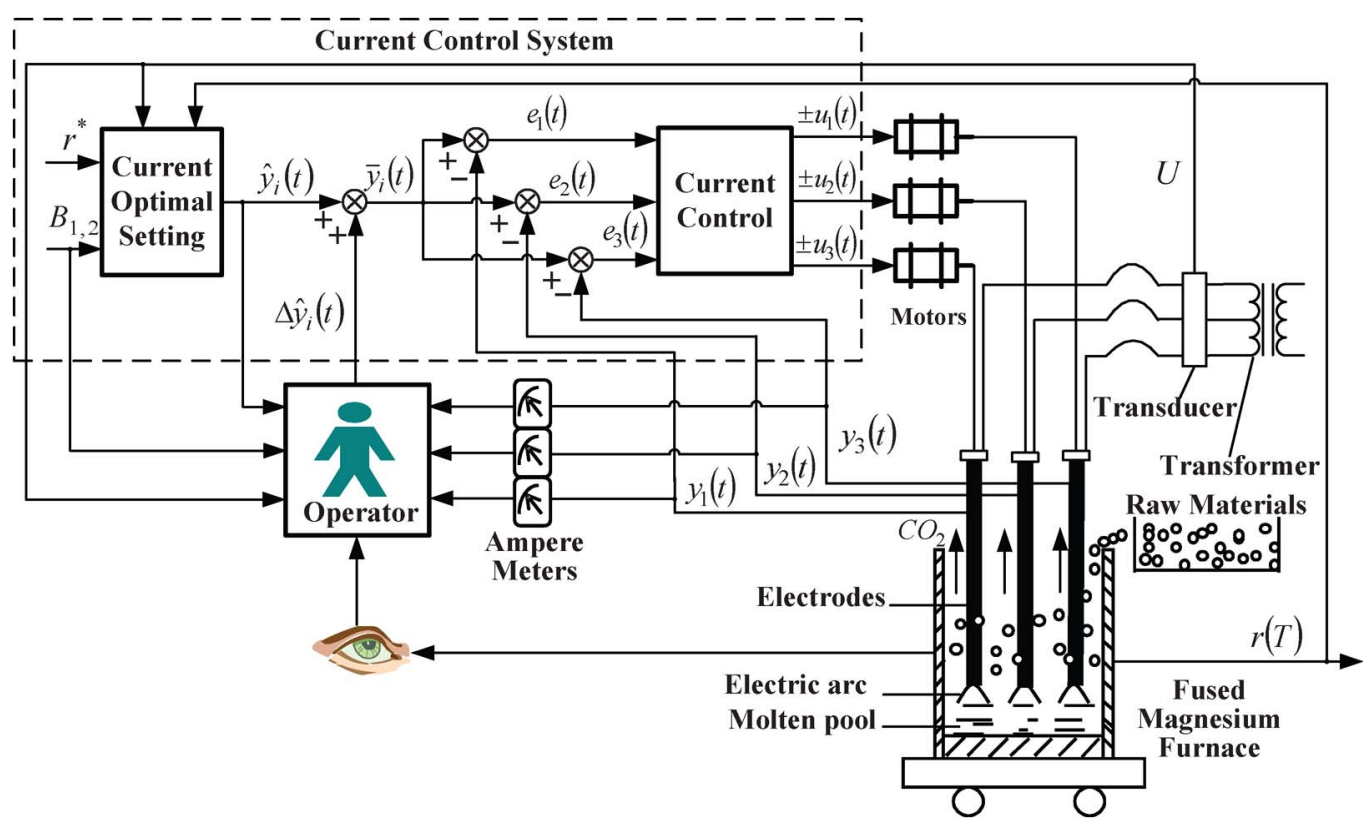

Fig. 2. Manual handling of abnormal conditions of the FMF.

Unfortunately, manual operation depends totally on human experience, and is susceptible to human errors. In this case, the abnormal conditions often cannot be identified or dealt with on time. Operating the smelting process under abnormal conditions for a long time period will lead to lower production rate, higher energy consumption and even a greater safety threat. Therefore, properly designed control strategies, which can replace the existing unreliable manual operation to identify and deal with abnormal conditions are of great interest.

\section{Data-Driven ABnormal Condition IDENTIFICATION AND SElF-healing CONTROL}

Abnormal conditions of the FMF smelting process happen when the system is operated at constant current setpoints $\hat{y}_{i}(t)$ for a sustained period of time when the arc resistances $R_{\text {arc }}^{a, b, c}$ are varied as consequences of changes in the raw material granule size $B_{1}$, the melting point $T_{\mathrm{mp}}$ and the resistivity $\rho_{\mathrm{rm}}$. Wherein, the changes in $T_{\mathrm{mp}}$ and $\rho_{\mathrm{rm}}$ are caused by the changes in raw material impurity constituents $B_{2}$. Therefore, the abnormal conditions can be expressed by the changes in $R_{\mathrm{arc}}^{a, b, c}$ and the duration $T_{i}(i=1, \ldots, 3)$. Note that $R_{\text {arc }}^{a, b, c}$ cannot be measured online, however the changes in $R_{\text {arc }}^{a, b, c}$ will cause variations in current tracking errors $E_{i}(t)$ and current change rates $\Delta y_{i}(t)$. Therefore, the abnormal conditions can be expressed as a function of $E_{i}(t), \Delta y_{i}(t)$ and duration $T_{i}$ as

$$
S_{L}(t)=f_{1}\left(E_{i}(t), \Delta y_{i}(t), T_{i}\right), \quad L=1, \ldots, 4
$$

where $S_{1}=S_{a}^{2}, S_{2}=S_{a}^{3}, S_{3}=S_{b}^{2}, S_{4}=S_{c}^{2}, E_{i}(t), \Delta y_{i}(t)$, and $T_{i}$ are given by

$$
E_{i}(t)=\frac{\sum_{j=1}^{3} y_{j}(t)}{3}-\hat{y}_{i}(t)
$$

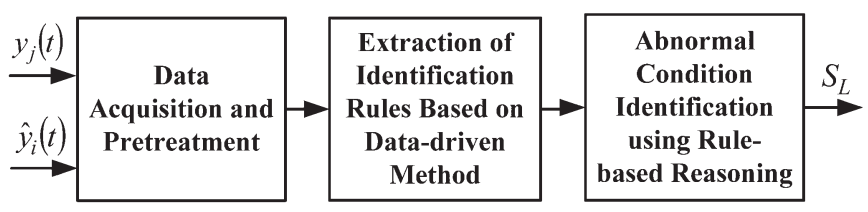

Fig. 3. Schematic of data-driven abnormal condition identification for the FMF.

$$
\begin{aligned}
\Delta y_{i}(t) & =\frac{\sum_{j=1}^{3}\left[y_{j}(t)-y_{j}(t-T)\right]}{3} \\
T_{i} & =n T
\end{aligned}
$$

where $i=1, \ldots, 3$ denote heating and melting, feeding, and exhausting conditions, respectively, $j=1, \ldots, 3$ denote the three-phase electrodes, and $T$ denotes the sampling period, $n$ denotes the integer multiple of the sampling period.

\section{A. Data-Driven Abnormal Condition Identification}

Combining the data of $T_{\mathrm{mp}}, \rho_{\mathrm{rm}}$ and $T_{i}$ with the analysis of the abnormal condition characteristics, a data-driven abnormal condition identification algorithm, consisting of a data acquisition and pretreatment unit, an identification rules extracting unit based on a data-driven method, and an RBR unit, is proposed in this paper as depicted in Fig. 3, and the realizations of the three units are introduced as follows:

1) Data Acquisition and Pretreatment: In order to remove the noise in the current data, the median absolute deviation filter [21] is used. First, the filtering method acquires $m$ current data $y_{j}^{m}$ at time $t(m=7)$ and sort the data according to the numerical value form the smallest to the largest, namely $y_{j}^{1}, y_{j}^{2}, \ldots, y_{j}^{4}, \ldots, y_{j}^{7}$, and then take the middle value $H^{M}=$ $y_{j}^{4}$. Second, the filtering method calculates the absolute values of the deviation $D_{j}^{k}=\left|y_{j}^{m}-H^{M}\right|(k=1, \ldots, 7)$, and sort 
TABLE I

Limited VALUES OF THE ANTECEDENT VARIABLES

\begin{tabular}{cc}
\hline Variables & Limited values \\
\hline$E_{i}(t)$ & $H_{E 1}, H_{E 2}, H_{E 3}$ \\
$\triangle y_{i}(t)$ & $H_{\triangle I 1}, H_{\triangle I 2}$ \\
$T_{i}$ & $t_{h}$ \\
\hline \hline
\end{tabular}

$D_{j}^{k}$ according to the numerical value form the smallest to the largest, $D_{j}^{1}, D_{j}^{2}, \ldots, D_{j}^{4}, \ldots, D_{j}^{7}$, to obtain the median value by taking $D=D_{j}^{4}$. Finally, the filtering method acquires the three-phase current data $\tilde{y}_{j}(t)$ at time $t$, and obtain the currents, which are used to identify the abnormal conditions by using

$$
y_{j}(t)=\left\{\begin{array}{lc}
\tilde{y}_{j}(t), & \text { if }\left|\tilde{y}_{j}(t)-H^{M}\right|<L \cdot M A D \\
H^{M}, & \text { else }
\end{array}\right.
$$

where $j=1, \ldots, 3, L$ denotes the threshold parameter $(L=$ $0.5), M A D=1.4826 \times D$. When the currents $y_{j}(t)$ are obtained, $E_{i}(t), \Delta y_{i}(t)$ and $T_{i}$ can be calculated using (10)-(12).

2) Extraction of Identification Rules Based on DataDriven Method: The abnormal condition identification uses rules in the form of "IF" \ promise > THEN" 〈 conclusion $>[22]-[25]$, wherein the premise represents the condition for the rule to be activated, and the conclusion is the abnormal condition corresponding to the premise. Considering the FMF smelting process characteristics, $E_{i}(t), \Delta y_{i}(t)$, and $T_{i}$ are used as antecedent variables of rule. The limited values of the antecedent variables in the entire range are listed in Table I.

Where $H_{E 1}, H_{E 2}, H_{E 3}$ are the lower limit, medium limit, and upper limit of the current tracking error $E_{i}(t)$, respectively. If $\left|E_{i}(t)\right|<H_{E 1}, H_{E 2}>\left|E_{i}(t)\right|>H_{E 1}, H_{E 3}>\left|E_{i}(t)\right|>$ $H_{E 2},\left|E_{i}(t)\right|>H_{E 3}$ represents the conditions that the current errors are within the target range, outside the target range but with small overshoots, relatively large, and very large, respectively. Similarly, $H_{\Delta I 1}, H_{\Delta I 2}$ denote the lower and upper limits of the current change rate $\Delta y_{i}(t)$. At last, $t_{h}$ denotes the threshold for the duration of a certain kind of current fluctuation. The aforementioned limited values are determined by the operator experience or by trial and error tests. Combining with the analysis of abnormal conditions, this paper extracts the rules from $E_{i}(t), \Delta y_{i}(t)$ and $T_{i}$ to identify the abnormal conditions $S_{L}(L=1, \ldots, 4)$.

a) Extraction of rules for semimolten condition $S_{1}$ : The increase in the melting point of raw material $T_{\mathrm{mp}}$ will cause raw materials that are not fully melted fall into the molten pools and the pool surfaces are raised. This leads to a decrease in the arc resistances $R_{\mathrm{arc}}^{a}$ and an increase in the currents $y_{j}(t)$. Since the current setpoints $\hat{y}_{1}(t)$ are unchanged, current tracking errors $E_{1}(t)$ are greater than the lower limit $H_{E 1}$, i.e., $E_{1}(t)>H_{E 1}$. At the same time, the raw materials falling into the molten pools will cause the surface fluctuation of molten pools, the absolute values of the change rates of the current $\Delta y_{1}(t)$ are larger than the lower limit $H_{\Delta I 1}$ and smaller than the higher limit $H_{\Delta I 2}$, i.e., $H_{\Delta I 2}>\left|\Delta y_{1}(t)\right|>H_{\Delta I 1}$. If the duration of the aforementioned current fluctuation $T_{1}$ is more than $t_{h}$, it will lead to the excessive accumulation of the gas in the molten pools, and the local areas of the furnace wall could be melted. Therefore, the related identification rule is expressed as

Rule 1: IF $E_{1}(t)>H_{E 1}$ AND $H_{\Delta I 2}>\left|\Delta y_{1}(t)\right|>H_{\Delta I 1}$ AND $T_{1}>t_{h}$ THEN $S_{1}$ will happen.

b) Extraction of rules for overheating condition $S_{2}$ : The decrease in the melting point of raw material $T_{m p}$ will cause the raw materials melting too fast, and the molten pool will rise faster than what is allowable. This will lead to a decrease in the arc resistances $R_{\mathrm{arc}}^{a}$ and a further increase in the currents $y_{j}(t)$. If the current setpoints $\hat{y}_{1}(t)$ are unchanged, the current tracking errors $E_{1}(t)$ will be positive and its value will be relatively or very large, i.e., $E_{1}(t)>H_{E 2}$. As the molten pool rises in a uniform speed when the raw materials are melt rapidly, small fluctuations of arc resistance $R_{\text {arc }}^{a}$ will lead to small change rate of the current $\Delta y_{1}(t)$, i.e., $\left|\Delta y_{1}(t)\right|<H_{\Delta I 1}$. If the duration of the aforementioned current fluctuation $T_{1}$ is more than $t_{h}$, impurities in the molten pool will not completely precipitate, which causes the degradation of product quality and the increase of energy consumption per ton. Therefore, the identification rule of the overheating condition can be expressed as

Rule 2: IF $E_{1}(t)>H_{E 2}$ AND $\left|\Delta y_{1}(t)\right|<H_{\Delta I 1}$ AND $T_{1}>$ $t_{h}$ THEN $S_{2}$ will happen.

c) Extraction of rules for abnormal feeding condition $S_{3}$ : During the feeding process, the increase in the resistivity of raw materials $\rho_{\text {rm }}$ can lead to a decrease in the currents $y_{j}(t)$. With unchanged current setpoints $\hat{y}_{2}(t)$, this will cause the current tracking errors $E_{2}(t)<0$, and the current controller will frequently adjust the electrode position down to track the current setpoints. The raw materials will slide into the molten pool directly, causing the fluctuation of surface of the molten pool. As a result, the arc resistance $R_{\text {arc }}^{b}$ will be unsteady and the absolute values of the change rate of the current $\Delta y_{2}(t)$ are large. Through the analysis of the feeding abnormal condition and the process data, there are three rules can be established as follows:

When the current tracking errors $E_{2}(t)$ are negative and very large, namely $E_{2}(t)<0$ and $\left|E_{2}(t)\right|>H_{E 3}$ and the duration of this current fluctuation $T_{2}$ exceeds $t_{h}$, the temperature distribution in the furnace will be disturbed by low temperature raw materials, regardless of the pattern in current change rate. This will lead to low product quality, and the identification rule for this case can be expressed as

Rule 3: IF $E_{2}(t)<0$ AND $\left|E_{2}(t)\right|>H_{E 3}$ AND $T_{2}>t_{h}$ THEN $S_{3}$ will happen.

When the current tracking errors $E_{2}(t)$ are negative and exceed the target range but with small overshoots, namely $E_{2}(t)<0$ and $H_{E 2}>\left|E_{2}(t)\right|>H_{E 1}$, a large amount of low temperature solid raw materials will fall into the molten pool, which will cause the surface of the molten pool to fluctuate. In this condition, the changes for arc resistance $R_{\text {arc }}^{b}$ increase, leading to a relatively large change rate of the current $\Delta y_{2}(t)$, 
namely $H_{\Delta I 2}>\left|\Delta y_{2}(t)\right|>H_{\Delta I 1}$. If the duration of this current fluctuation exceeds the threshold $T_{2}>t_{h}$, the temperature distribution in the furnace will be also disturbed by low temperature raw materials, leading to low product quality. Therefore, the extraction rules can be expressed as

Rule 4: IF $E_{2}(t)<0$ AND $H_{E 2}>\left|E_{2}(t)\right|>H_{E 1}$ AND $H_{\Delta I 2}>\left|\Delta y_{2}(t)\right|>H_{\Delta I 1} \quad$ AND $T_{2}>t_{h}$ THEN $S_{3}$ will happen.

Finally, when the current tracking errors $E_{2}(t)$ are negative and relatively large, namely $E_{2}(t)<0$ and $H_{E 3}>\left|E_{2}(t)\right|>$ $H_{E 2}$, if a small amount of low temperature solid raw materials evenly fall into the molten pool, it will cause the surface of the molten pool sloshing slightly. In this case, the change rates of arc resistance $R_{\mathrm{arc}}^{b}$ and current are small, namely $\left|\Delta y_{2}(t)\right|<$ $H_{\Delta I 1}$. If the duration of this current fluctuation $T_{2}$ is more than $t_{h}$, the uniform temperature distribution in the furnace will be also destroyed by low temperature raw materials. Therefore, the extraction rules can be expressed as

Rule 5: IF $E_{2}(t)<0$ AND $H_{E 3}>\left|E_{2}(t)\right|>H_{E 2}$ AND $\left|\Delta y_{2}(t)\right|<H_{\Delta I 1}$ AND $T_{2}>t_{h}$ THEN $S_{3}$ will happen.

d) Extraction of rules for abnormal exhausting condition $S_{4}$ : During the exhausting process, when there is a substantial change in the raw material particle size $B_{1}$ the gaps between the raw materials and the electrodes will not be appropriate and the pressure inside the FMF cannot be balanced with the atmospheric pressure if the current setpoints $\hat{y}_{3}(t)$ are unchanged. In this case, under the influence of air pressure, the fluctuations of the gap between the surface of the molten pool and threephase electrode cause large changes in arc resistance $R_{\mathrm{arc}}^{c}$. Through the analysis of the exhausting abnormal condition and the process data, rules of identifying abnormal exhausting condition are as follows:

When the surface of the molten pool rises, the arc length reduces, and the arc resistance $R_{\mathrm{arc}}^{c}$ decreases, thereby leading to an increase in the smelting currents beyond the target range. In this case, the current tracking errors $E_{3}(t)$ are positive and bigger than the lower limit, namely, $E_{3}(t)>H_{E 1}$. At the same time, the shakes of an electrode cause the arc resistance $R_{\mathrm{arc}}^{c}$ to fluctuate in a large range, the absolute values of change rates of the currents are very large, namely $\left|\Delta y_{3}(t)\right|>H_{\Delta I 2}$. If the duration of this current fluctuation exceeds the threshold $T_{3}>$ $t_{h}$, the high-temperature melts can spurt out of the furnace. The identification rule of the abnormal exhausting condition can be expressed as

Rule 6: IF $E_{3}(t)>H_{E 1} \operatorname{AND}\left|\Delta y_{3}(t)\right|>H_{\Delta I 2} \operatorname{AND} T_{3}>$ $t_{h}$ THEN $S_{4}$ will happen.

When the surface of the molten pool is reduced, the arc resistance $R_{\mathrm{arc}}^{c}$ will increase and the currents $y_{j}(t)$ will decrease, the current tracking errors $E_{3}(t)$ are positive and relatively large, namely $E_{3}(t)<0, H_{E 3}>\left|E_{3}(t)\right|>H_{E 2}$. At the same time, the shakes of electrode cause the relatively or very large change of current, namely $\left|\Delta y_{3}(t)\right|>H_{\Delta I 1}$. If the duration of
TABLE II

RULES FOR ABNORMAL CONDITION IDENTIFICATION

\begin{tabular}{|c|c|c|}
\hline Rules & Antecedents & Conclusions \\
\hline 1 & $\begin{array}{c}E_{1}(t)>H_{E 1} \text { AND } \\
H_{\triangle I 2}>\left|\triangle y_{1}(t)\right|>H_{\triangle I 1} \text { AND } T_{1}>t_{h}\end{array}$ & $S_{1}$ \\
\hline 2 & $\begin{array}{c}E_{1}(t)>H_{E 2} \text { AND }\left|\triangle y_{1}(t)\right|<H_{\triangle I 1} \text { AND } \\
T_{1}>t_{h}\end{array}$ & $S_{2}$ \\
\hline 3 & $\begin{array}{c}E_{2}(t)<0 \text { AND }\left|E_{2}(t)\right|>H_{E 3} \text { AND } \\
T_{2}>t_{h}\end{array}$ & $S_{3}$ \\
\hline 4 & $\begin{array}{c}E_{2}(t)<0 \text { AND } H_{E 2}>\left|E_{2}(t)\right|>H_{E 1} \text { AND } \\
H_{\triangle I 2}>\left|\triangle y_{2}(t)\right|>H_{\triangle I 1} \text { AND } T_{2}>t_{h}\end{array}$ & $S_{3}$ \\
\hline 5 & $\begin{array}{c}E_{2}(t)<0 \text { AND } H_{E 3}>\left|E_{2}(t)\right|>H_{E 2} \text { AND } \\
\left|\triangle y_{2}(t)\right|<H_{\triangle I 1} \text { AND } T_{2}>t_{h}\end{array}$ & $S_{3}$ \\
\hline 6 & $\begin{array}{c}E_{3}(t)>H_{E 1} \text { AND }\left|\triangle y_{3}(t)\right|>H_{\triangle I 2} \text { AND } \\
T_{3}>t_{h}\end{array}$ & $S_{4}$ \\
\hline 7 & $\begin{array}{c}E_{3}(t)<0 \text { AND } H_{E 3}>\left|E_{3}(t)\right|>H_{E 2} \text { AND } \\
\left|\triangle y_{3}(t)\right|>H_{\triangle I 1} \text { AND } T_{3}>t_{h}\end{array}$ & $S_{4}$ \\
\hline 8 & $\begin{array}{c}E_{3}(t)<0 \text { AND } H_{E 2}>\left|E_{3}(t)\right|>H_{E 1} \text { AND } \\
\left|\triangle y_{3}(t)\right|>H_{\triangle I 2} \text { AND } T_{3}>t_{h}\end{array}$ & $S_{4}$ \\
\hline
\end{tabular}

this current fluctuation exceeds its threshold $T_{3}>t_{h}$, the hightemperature melts can spurt out of the furnace. Therefore, the extraction rules can be expressed as

Rule 7: IF $E_{3}(t)<0$ AND $H_{E 3}>\left|E_{3}(t)\right|>H_{E 2}$ AND $\left|\Delta y_{3}(t)\right|>H_{\Delta I 1}$ AND $T_{3}>t_{h}$ THEN $S_{4}$ will happen.

When the surface of the molten pool reduces, the arc resistance $R_{\mathrm{arc}}^{c}$ will increase and the currents $y_{j}(t)$ will decrease. The current tracking errors $E_{3}(t)$ are positive and exceed the target range but with small overshoots, namely $E_{3}(t)<$ $0, H_{E 2}>\left|E_{3}(t)\right|>H_{E 1}$. At the same time, the shakes of electrode cause the arc resistance $R_{\mathrm{arc}}^{c}$ fluctuate in a large range, leading to the very large changes in currents, namely $\left|\Delta y_{3}(t)\right|>H_{\Delta I 2}$. If the duration of this current fluctuation exceeds its threshold $T_{3}>t_{h}$, the high-temperature melts can also spurt out of the furnace similarly. Therefore, the extraction rules can be expressed as

Rule 8: IF $E_{3}(t)<0$ AND $H_{E 2}>\left|E_{3}(t)\right|>H_{E 1}$ AND $\left|\Delta y_{3}(t)\right|>H_{\Delta I 2}$ AND $T_{3}>t_{h}$ THEN $S_{4}$ will happen.

\section{3) RBR System for Abnormal Conditions Identification:}

Based on the aforementioned process, the identification rules can be extracted, and the rule base for abnormal condition identification is shown in Table II.

The current tracking errors, the change rates of the current, and the duration of current fluctuation are used to match the antecedents in the rule base, and the specific abnormal condition is the reasoning result based on the forward reasoning mechanism. The reasoning procedure is shown in Fig. 4 and is introduced as follows:

Step 1) Initialization, make $z=1$. Calculate the $E_{i}(t)$, $\Delta y_{i}(t)$ and $T_{i}$ by using (10)-(12).

Step 2) Determine whether $E_{i}(t), \Delta y_{i}(t)$ and $T_{i}$ at time $t$ can match the antecedents in rule or not. If the data can match the antecedents, then the reasoning procedure outputs the type of the corresponding abnormal condition $(S=1, \ldots, 4$ denote the current condition is $S_{1}-S_{4}$, respectively). If the data cannot match 


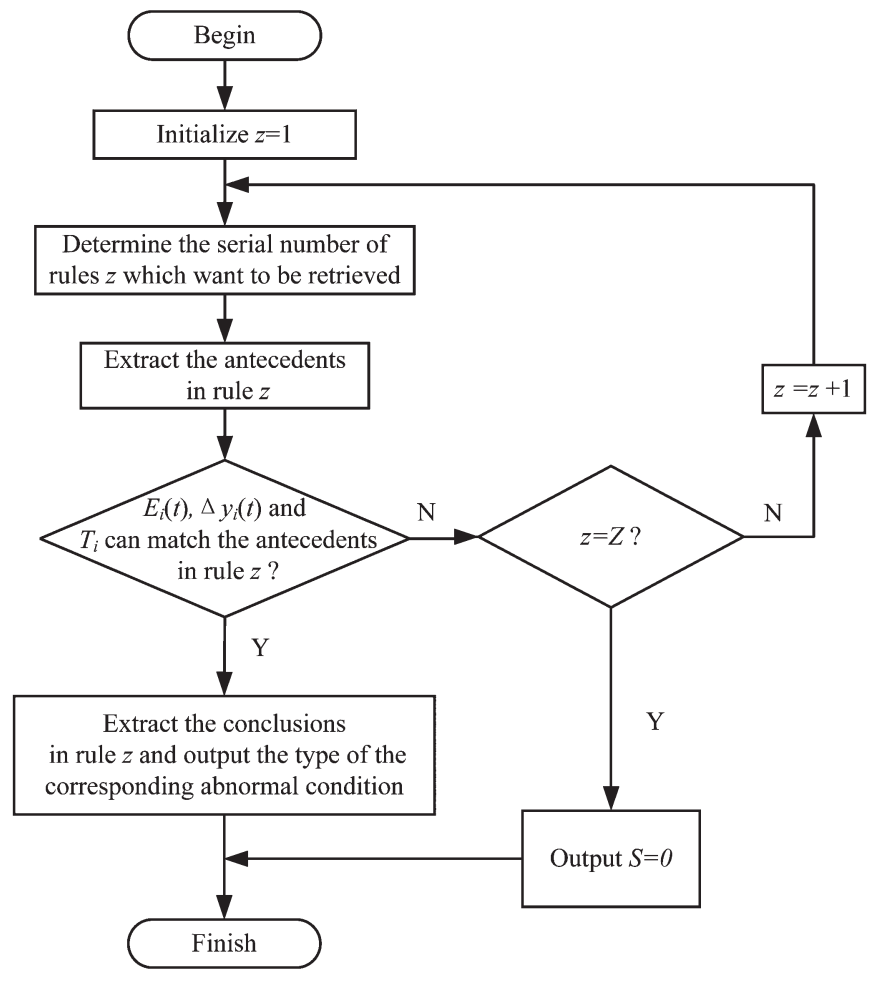

Fig. 4. Forward reasoning mechanism for RBR.

the antecedents, then the reasoning procedure turns to Step 4, namely,

$$
\begin{aligned}
& \text { if } E_{1}(t)>H_{E 1}, H_{\Delta I 2}>\left|\Delta y_{1}(t)\right|>H_{\Delta I 1}, \\
& T_{1}>t_{h} \text { then } S=1 \\
& \text { elseif } E_{1}(t)>H_{E 2},\left|\Delta y_{1}(t)\right|<H_{\Delta I 1}, \\
& T_{1}>t_{h} \text { then } S=2 \\
& \text { elseif } E_{2}(t)<0,\left|E_{2}(t)\right|>H_{E 3}, T_{2}>t_{h} \text { or } \\
& E_{2}(t)<0, H_{E 2}>\left|E_{2}(t)\right|>H_{E 1}, \\
& \quad H_{\Delta I 2}>\left|\Delta y_{2}(t)\right|>H_{\Delta I 1}, T_{2}>t_{h} \text { or } \\
& \quad E_{2}(t)<0, H_{E 3}>\left|E_{2}(t)\right|>H_{E 2}, \\
& \quad\left|\Delta y_{2}(t)\right|<H_{\Delta I 1}, T_{2}>t_{h} \text { then } S=3 \\
& \text { elseif } E_{3}(t)>H_{E 1},\left|\Delta y_{3}(t)\right|>H_{\Delta I 2}, \\
& \quad T_{3}>t_{h} \text { or } E_{3}(t)<0, H_{E 3}>\left|E_{3}(t)\right|>H_{E 2}, \\
& \left|\Delta y_{3}(t)\right|>H_{\Delta I 1}, T_{3}>t_{h} \text { or } E_{3}(t)<0, \\
& H_{E 2}>\left|E_{3}(t)\right|>H_{E 1},\left|\Delta y_{3}(t)\right|>H_{\Delta I 2}, \\
& T_{3}>t_{h} \text { then } S=4 \\
& \text { end if }
\end{aligned}
$$

Step 3) Determine if $z=Z$ is satisfied, where $Z$ is the number of rules in the rule base. If $z=Z$, then the reasoning procedure output is $S=0(S=0$ denotes the current condition is normal condition) and turns to Step 4. If $z \neq Z$, then the reasoning procedure makes $z=z+1$, and turns to Step 2 .

Step 4) Finish the reasoning procedure.

\section{B. Self-Healing Control Based on CBR}

Based on the aforementioned data-driven abnormal condition identification method, a strategy for abnormal condition iden- tification and self-healing control is proposed in this paper as depicted in Fig. 5.

Given that the abnormal conditions of the FMF smelting process are typically caused by improperly defined setpoints of the current, the CBR-based self-healing controller is used to calculate the correction of current setpoints $\Delta \hat{y}_{i}(t)$ when the abnormal conditions occur. The outputs of the current controller track the corrected setpoints $\bar{y}_{i}(t)=\hat{y}_{i}(t)+\Delta \hat{y}_{i}(t)$, which makes the FMF smelting process recover gradually from the abnormal conditions.

The corrected values $\Delta \hat{y}_{i}(t)$ for the current setpoints to heal the abnormal conditions are closely related to currents $y_{j}(t)$, current setpoints $\hat{y}_{i}(t)$, types of abnormal condition $S_{L}$, smelting voltage $U$, raw material granule size $B_{1}$ and raw material impurity constituent $B_{2}$, its functional can be described as follows:

$$
\Delta \hat{y}_{i}(t)=f_{2}\left(y_{j}(t), \hat{y}_{i}(t), S_{L}, U, B_{1}, B_{2}\right)
$$

where $f_{2}(\cdot)$ is an unknown nonlinear function. The relationship between $\Delta \hat{y}_{i}(t), B_{1}$, and $B_{2}$ has very complicated dynamic characteristics that involve nonlinearities, couplings among subsystems and persistent random disturbances. In general, it is very difficult to obtain accurate models for $f_{2}(\cdot)$ because these models vary in a wide range along with dynamical characteristics of the system. As such, it is difficult to design and implement model-based adjustment algorithm using available methodologies and tools.

Therefore, a self-healing control, as shown in Fig. 6, is constructed in this paper with CBR technique [26]-[31]. The key elements of the control scheme are described as follows:

Case representation and case retrieval: According to the expert experiences, the initial cases are obtained and denoted by $y_{j}(t), \hat{y}_{i}(t), S_{L}, U, B_{1}$, and $B_{2}$. The case solution $y_{s k}$ is presented by the correction of current setpoints $\Delta \hat{y}_{i}(t)$. The case structure is shown in Table III, where $c_{1}, \ldots, c_{5}$ denote the three-phase electrode currents, current setpoint, and smelting voltage, respectively, $c_{6}$ denotes the abnormal conditions and takes values in set $\{1,2,3,4\} . c_{7}$ and $c_{8}$ denote the raw materials granule size $B_{1}$ and raw materials impurity constituent $B_{2}$. The granule size and impurity constituent of raw materials cannot be measured online, and can only be described qualitatively. Therefore, $c_{7}$ and $c_{8}$ take values in set $\{1,2,3\}$, and $1,2,3$ for $B_{1}$ represents the granule size of the raw material being big, medium, small, and $B_{2}$ represents the impurity constituent of raw material being good, medium, poor, respectively.

The reasoning system searches and matches the case according to the characteristics of smelting process. The characteristics of the current condition $M$ is described as $C=\left\{c_{l}\right\}, l=1, \ldots, 8$, and the solution of $M$ is the $y_{s k}=$ $\Delta \hat{y}_{i}(t)$. The $k$ th case $M_{k}$ in the case-base is described as $C_{k}=\left\{c_{l, k}\right\}, k=1, \ldots, K$ and the solution of $C_{k}$ is the $y_{s k, k}=\Delta \hat{y}_{i, k}(t)$, where $K$ is the number of cases. The similarity between $c_{1}, \ldots, c_{5}$ and $c_{l, k}$ are given by

$$
\operatorname{sim}\left(c_{l}, c_{l, k}\right)=1-\frac{\left|c_{l}-c_{l, k}\right|}{\max \left(c_{l}, c_{l, k}\right)}, \quad \text { when } l=1, \ldots, 5
$$




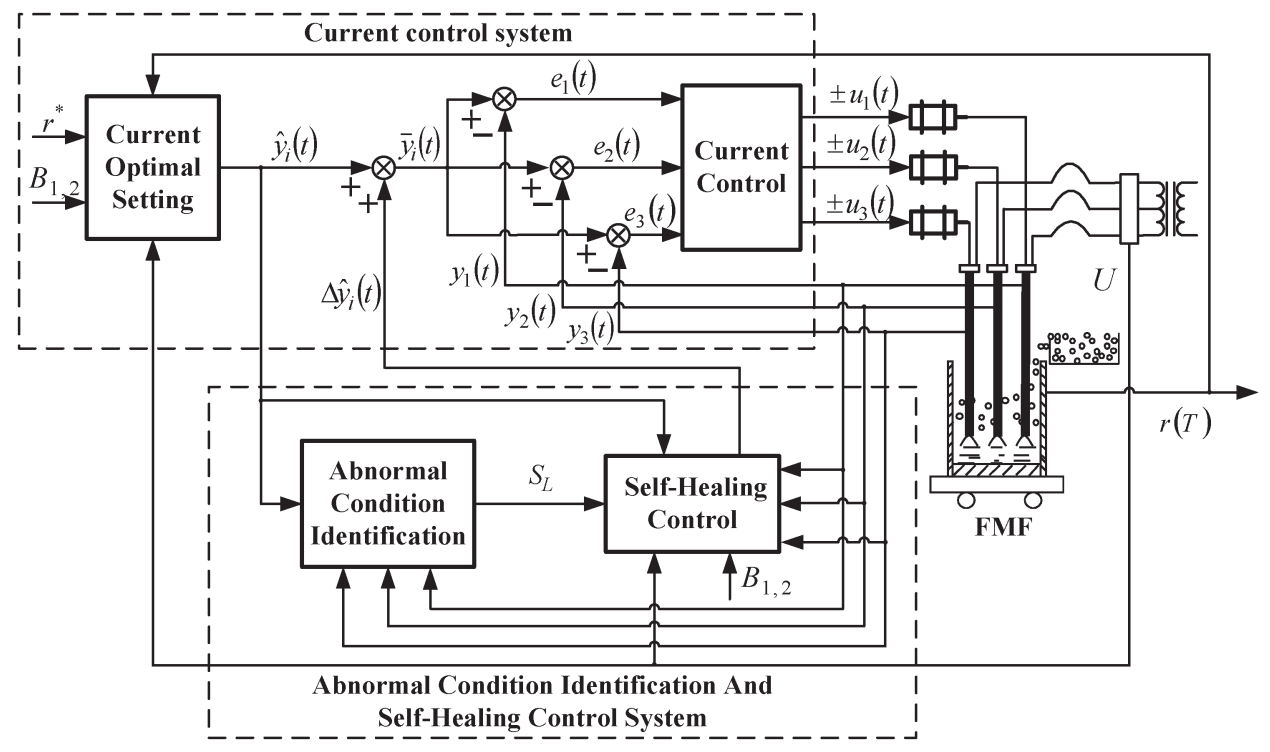

Fig. 5. Abnormal condition identification and self-healing control strategy for the FMF.

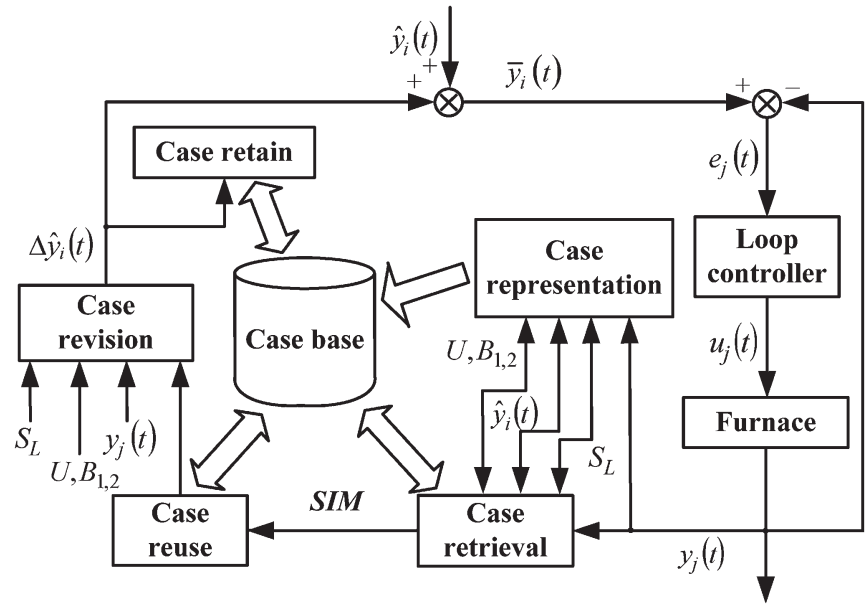

Fig. 6. CBR based self-healing controller.

TABLE III

The Case Structure for Self-Healing Controller

\begin{tabular}{ccccccccc}
\hline \hline \multicolumn{9}{c}{ Case Description } \\
\hline$y_{1}(t)$ & $y_{2}(t)$ & $y_{3}(t)$ & $\hat{y}_{i}(t)$ & $U$ & $S_{L}$ & $B_{1}$ & $B_{2}$ & Case Solution \\
$c_{1}$ & $c_{2}$ & $c_{3}$ & $c_{4}$ & $c_{5}$ & $c_{6}$ & $c_{7}$ & $c_{8}$ & \\
\hline \hline
\end{tabular}

The similarity between the abnormal condition $c_{6}$ and $c_{l, k}$ is given by

$$
\operatorname{sim}\left(c_{6}, c_{l, k}\right)= \begin{cases}1, & c_{6}=c_{l, k} \\ 0.8, & \left|c_{6}-c_{l, k}\right|=1 \\ 0.7, & \left|c_{6}-c_{l, k}\right|=2 \\ 0.6, & \left|c_{6}-c_{l, k}\right|=3\end{cases}
$$

The similarity between granule size of raw materials $c_{7}$ and $c_{l, k}$ is given by

$$
\operatorname{sim}\left(c_{7}, c_{l, k}\right)= \begin{cases}1, & c_{7}=c_{l, k} \\ 0.8, & \left|c_{7}-c_{l, k}\right|=1 \\ 0.6, & \left|c_{7}-c_{l, k}\right|=2 .\end{cases}
$$

The similarity between the impurity constituent of raw materials $c_{8}$ and $c_{l, k}$ is given by

$$
\operatorname{sim}\left(c_{8}, c_{l, k}\right)= \begin{cases}1, & c_{8}=c_{l, k} \\ 0.65, & \left|c_{8}-c_{l, k}\right|=1 \\ 0.2, & \left|c_{8}-c_{l, k}\right|=2 .\end{cases}
$$

The similarity between the current condition $M$ and $M_{k}$ is given by

$$
\operatorname{SIM}\left(M, M_{k}\right)=\frac{\sum_{l=1}^{8} \lambda_{l} \operatorname{sim}\left(c_{l}, c_{l, k}\right)}{\sum_{l=1}^{8} \lambda_{l}}
$$

where $\lambda_{l}$ denote the weights of cases which are determined by experience and trial and error method.

Case reuse: The retrieved cases cannot be directly used to adjust the current setpoint because the cases from case-base are not completely matched with the current condition. Thus, the case reuse unit calculates the case solution $\Delta \hat{y}_{i}(t)$ for current condition $M$ using

$$
\Delta \hat{y}_{i}(t)=\frac{\sum_{k=1}^{K} \operatorname{SIM}\left(M, M_{k}\right) \times \Delta \hat{y}_{i, k}(t)}{\sum_{k=1}^{K} \operatorname{SIM}\left(M, M_{k}\right)} .
$$

Case revision and retain: If the abnormal conditions can be removed with the case solution $\Delta \hat{y}_{i}(t)$, the case would be saved. If the abnormal conditions could not be completely removed, the algorithm will adjust the setpoint until the abnormal conditions are completely removed and the revised case will be saved. The algorithm determines whether it is needed to add the case to the case-base according to the characteristics of the smelting process $M$ and the similarity between the $M$ and existing cases in the case-base. Thus, if these characteristics (raw material granule sizes, raw material impurity constituents, and working conditions) 
TABLE IV

PARAMETERS OF PRODUCTION EQUIPMENT

\begin{tabular}{cc}
\hline \hline graphite electrode diameter & $350 \mathrm{~mm}$ \\
rated voltage & $110 \mathrm{~V}$ \\
shell diameter & $2.5 \mathrm{~m}$ \\
rated current & $13-17 \mathrm{KA}$ \\
rated smelting time & $10 \mathrm{~h}$ \\
\hline \hline
\end{tabular}

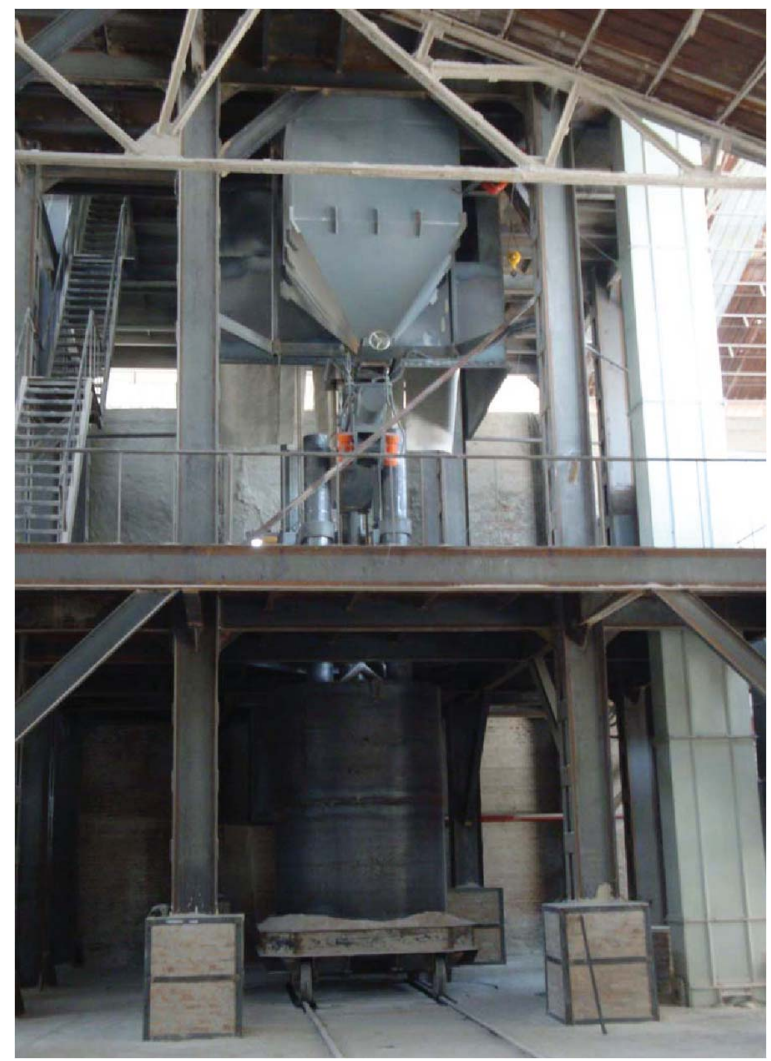

Fig. 7. FMF.

are greatly different from those cases in the case-base, and maximum similarity between state $M$ and the existing cases in the case-base is less than the reference threshold, the case $M$ will be considered as a new case and added to the case-base.

\section{INDUSTRIAL APPLICATION}

The proposed method is applied to a real fused magnesia factory in China. This factory annually produces around 100000 tons of all types of fused magnesia. The parameters of production equipment are shown in Table IV.

The abnormal condition identification and self-healing control described in Section III is adapted and applied for the FMF smelting process shown in Fig. 7. The hardware structure of the control system is shown in Fig. 8. The current loop controller is designed using the method in [1], and the method in [2] is used to generate the current setpoints for the normal smelting process.

The limit values of the antecedent variables in the abnormal condition identification rules are determined by operator experience. When $E_{i}(t)>0, H_{E 1}=1500 \mathrm{~A}, H_{E 2}=2000 \mathrm{~A}$, $H_{E 3}=2500 \mathrm{~A}$; when $E_{i}(t)<0, H_{E 1}=1500 \mathrm{~A}, H_{E 2}=$

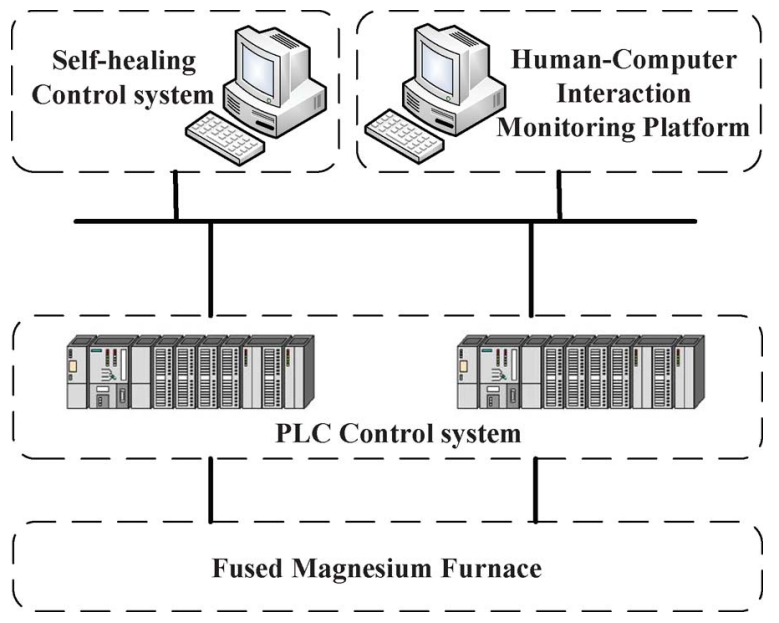

Fig. 8. Hardware structure for control system.

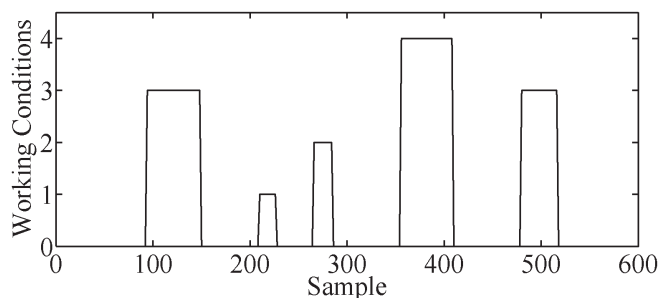

(a)

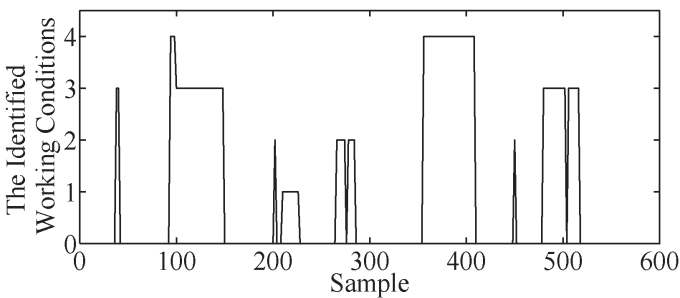

(b)

Fig. 9. Comparison of results of abnormal condition identification. (a) The actual working conditions; (b) the effect of working conditions recognition.

$3000 \mathrm{~A}, H_{E 3}=5000 \mathrm{~A}$. The limiting values of $\Delta y_{i}(t)$ and $T_{i}$ are obtained by trial and error tests. In order to verify the selection of the limit values, 600 groups of actual process data are used. When $H_{\Delta I 1}=1200 \mathrm{~A}, H_{\Delta I 1}=2000$ Aand $t_{h}=$ $30 \mathrm{~s}$, the result is shown in Fig. 9, where (a) shows the actual smelting conditions and (b) shows the identification result of the abnormal conditions. In order to simplify the presentation, in Fig. 9, $S=0,1,2,3,4$ represent the normal, semimolten, overheating, abnormal feeding, and abnormal exhausting condition, respectively. One can see that the identification algorithm correctly identifies the operating condition in most cases with the false alarm rate about $7 \%$. In view of this result, the limit values of antecedent variables aforementioned are appropriate.

The responses of the electrical currents (three different colors represent the currents for the three phase electrodes) with manual operation are shown as Fig. 10. It can be seen that the currents cannot track the setpoints very well and they vary over a wide. It indicates that manually adjusting the setpoints cannot guarantee a satisfactory smelting process. 


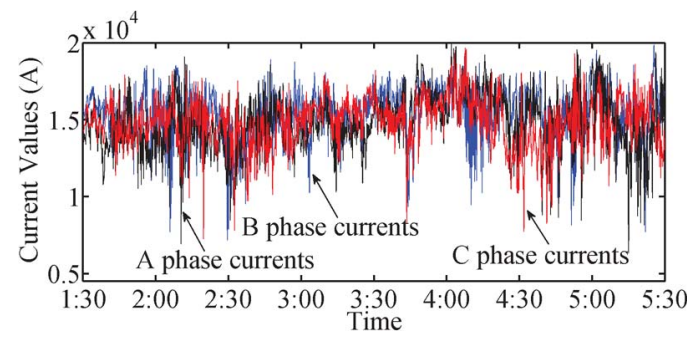

Fig. 10. Abnormal condition control effect for electrical currents with manual adjusts the setpoint.
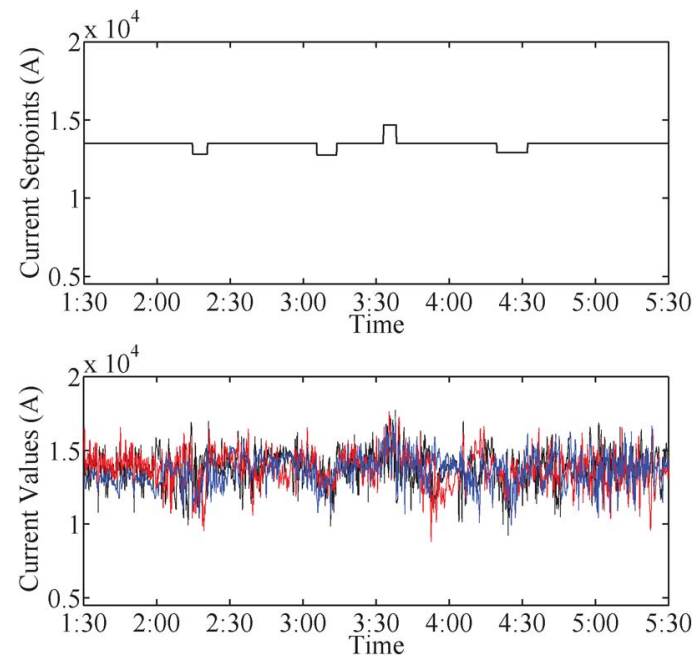

Fig. 11. Setpoint and control effect for electrical currents with proposed method.

TABLE V

Operating Points of the Smelting Process

\begin{tabular}{cccccc}
\hline \hline Time & $1: 30$ & $2: 12$ & $3: 06$ & $3: 33$ & $4: 24$ \\
\hline$E_{i}(t)$ & 912.42 & 1700.55 & 2175.83 & 1842.79 & -3534.78 \\
$\triangle y_{i}(t)$ & 1005.67 & 2146.73 & 763.87 & -1476.46 & 1037.76 \\
$\hat{y}_{i}(t)$ & 13500 & 13100 & 13500 & 13500 & 12500 \\
$U$ & 110 & 110 & 110 & 110 & 110 \\
$S$ & 0 & 4 & 2 & 1 & 3 \\
$B_{1}$ & 1 & 1 & 1 & 1 & 3 \\
$B_{2}$ & 1 & 1 & 2 & 3 & 3 \\
$\triangle \hat{y}_{i}(t)$ & 0 & -282 & -736 & 1176 & 405 \\
$\bar{y}_{i}(t)$ & 13500 & 12818 & 12764 & 14676 & 12905 \\
\hline \hline
\end{tabular}

With the proposed algorithm, the current setpoints and the responses of the electrical currents are shown in Fig. 11, and the operating points of the smelting process are shown in Table V.

During 1:30 A.M. $-2: 12$ A.M., the smelting process is in normal condition. At 2:12 A.M., the gas produced during the smelting process needs to be displaced. The current setpoint was adjusted to $\hat{y}_{3}(2: 12)=13100 \mathrm{~A}$. The tracking error $E_{3}(2: 12)=1700.55 \mathrm{~A}$ is larger than the target range $[-1500 \mathrm{~A}, 1500 \mathrm{~A}]$, and the current change rate $\Delta y_{3}(2: 12)=$ $2146.73 \mathrm{~A}$ is very large and the duration lasts more than $30 \mathrm{~s}$. Following the identification rules, the algorithm detects the abnormal exhausting condition $S_{4}$.

The self-healing controller adopts the aforementioned identification result and executes CBR. The case description of the current working condition $M$ is shown in Table VI.
TABLE VI

Case Description for Self-Healing Controller

\begin{tabular}{cccccccc}
\hline \hline \multicolumn{10}{c}{ Case Description } \\
\hline$c_{1}$ & $c_{2}$ & $c_{3}$ & $c_{4}$ & $c_{5}$ & $c_{6}$ & $c_{7}$ & $c_{8}$ \\
13781.25 & 16002.33 & 14618.06 & 13100 & 110 & 4 & 1 & 1 \\
\hline \hline
\end{tabular}

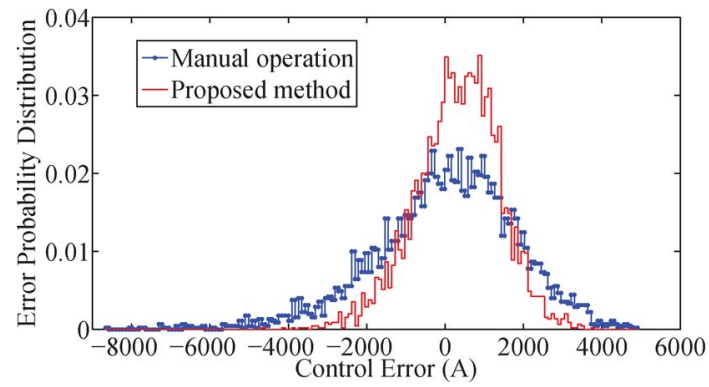

Fig. 12. Tracking error probability distribution.

Using (19), the similarity between $M$ and the stored case $M_{k}$ is given by

$\operatorname{SIM}\left(M, M_{k}\right)=\{0.823,0.815,0.803,0.826,0.796,0.752$,

$0.828,0.755,0.821\}$

where the weight of each feature is determined as follows:

$$
\begin{aligned}
\lambda & =\left\{\lambda_{1}, \ldots, \lambda_{8}\right\} \\
& =\{0.85,0.85,0.85,0.71,0.75,0.65,0.64,0.68\} .
\end{aligned}
$$

According to $\operatorname{SIM}\left(M, M_{k}\right)$, the case solution at the time 2:12 A.M. can be determined as $\Delta \hat{y}_{3}(2: 12)=-282 \mathrm{~A}$. Therefore, the setpoint is adjusted to $\bar{y}_{3}(2: 12)=13100-282=$ 12818 A. The control system tracks the corrected setpoint and at 2:16 A.M. and the smelting process recovers to normal condition.

At 3:06 A.M., the impurity constituent of raw materials $B_{2}$ changes from "good" to "medium", leading to the overheating abnormal condition $S_{2}$. This condition is identified, and the current setpoint at this time is adjusted to $\bar{y}_{3}(3: 06)=$ $13500-736=12764$ according to the self-healing algorithm. At 3:11A.M., the smelting process exits $S_{2}$ and is back to the normal condition. At 3:33 A.M. and 4:24 A.M., the working conditions change again, and the operating parameters are shown in Table V.

The tracking error probability distribution with the proposed method and the manual operation are shown in Fig. 12. The current tracking errors with proposed method are controlled to be within acceptable range $[-1500 \mathrm{~A}, 1500 \mathrm{~A}]$ for $83 \%$ of the time, whereas it is $48 \%$ for the manual operation. This shows that the proposed control strategy significantly improves the performance compared with manual operation.

The performance improvement of the proposed abnormal condition identification and self-healing control system has been confirmed through extensive testing. The factory application results of the proposed system have shown that the occurrence of abnormal condition is reduced by over $50 \%$, the product quality is increased by more than $2 \%$, and the power consumption is decreases by more than $3 \%$. 


\section{CONCLUSION}

The abnormal conditions occur frequently and are difficult to identify and deal with in the FMF smelting process. To solve this problem, this paper presents a data-driven abnormal condition identification and self-healing control system. The RBR-based abnormal condition identification algorithm extract the rules using the data of current tracking error, change rate of the current and duration for currents fluctuation, and then identifies the abnormal conditions during the heating and melting, feeding, and exhausting conditions. If the abnormal conditions are detected, the CBR-based self-healing controller adjusts the current setpoints automatically according to the currents, current setpoints, production equipment parameters, raw material parameters and the type of abnormal conditions. Once the current control system achieves tracking of the revised setpoints, the smelting process recovers from the diagnosed abnormal conditions. The successful application in the FMF smelting process shows that the proposed method provides a new solution for fault diagnosis and fault cancellation of complex industrial processes with substantial benefits in improving product quality and reducing cost.

\section{REFERENCES}

[1] Z. W. Wu, Y. J. Wu, and T. Y. Chai, "Intelligent control of fused magnesium furnaces based on SPSA," J. Shang Hai JiaoTong Univ., vol. 45, no. 8, pp. 1096-1100, Aug. 2011.

[2] Y. J. Wu, L. Zhang, H. Yue, and T. Y. Chai, "Intelligent optimal control based on CBR for fused magnesia production," J. Chem. Ind. Eng. (China), vol. 159, no. 7, pp. 1686-1690, Jul. 2008.

[3] Z. W. Wu, T. Y. Chai, J. Fu, and J. Sun, "The hybrid intelligent optimization control for the fused magnesium furnace," in Proc. IEEE Conf. Decis. Control, 2010, pp. 3313-3318.

[4] A. Y. Goharrizi and N. Sepehri, "A wavelet-based approach for external leakage detection and isolation from internal leakage in valve-controlled hydraulic actuators," IEEE Trans. Ind. Electron., vol. 58, no. 9, pp. $4374-$ 4384, Sep. 2011

[5] B. Zhang et al., "A probabilistic fault detection approach: Application to bearing fault detection," IEEE Trans. Ind. Electron., vol. 58, no. 5, pp. 2011-2018, May 2011.

[6] S. Yin, L. Hao, and S. X. Ding, "Real-time implementation of faulttolerant control systems with performance optimization," IEEE Trans. Ind. Electron., vol. 61, no. 5, pp. 2402-2411, May 2014.

[7] S. Yin, S. X. Ding, A. Haghani, H. Y. Hao, and P. Zhang, "A comparison study of basic data-driven fault diagnosis and process monitoring methods on the benchmark Tennessee Eastman process," J. Process Control, vol. 22, no. 9, pp. 1567-1581, Oct. 2012.

[8] Q. Liu, S. J. Qin, and T. Y. Chai, "Decentralized fault diagnosis of continuous annealing processes based on multilevel PCA," IEEE Trans. Autom. Sci. Eng., vol. 10, no. 3, pp. 687-698, Jul. 2013.

[9] D. Li, B. S. Liu, S. J. Qin, and D. H. Zhou, "Quality relevant datadriven modeling and monitoring of multivariate dynamic processes: The dynamic T-PLS approach," IEEE Trans. Neural Netw., vol. 22, no. 12, pp. 2262-2271, Dec. 2011.

[10] Y. W. Zhang and S. Li, "Modeling and monitoring between-mode transition of multimodes processes," IEEE Trans. Ind. Informat., vol. 9, no. 4, pp. 2248-2255, Nov. 2013.

[11] Y. W. Zhang, J. Y. An, Z. M. Li, and H. Wang, "Modeling and monitoring for handling nonlinear dynamic processes," Inf. Sci., vol. 235, no. 6, pp. 97-105, Jun. 2013.

[12] H. You, V. Vittal, and Z. Yang, "Self-healing in power system: An approach using islanding and rate of frequency decline-based load shedding," IEEE Trans. Power Syst., vol. 18, no. 1, pp. 174-181, Feb. 2003.

[13] K. Seethalekshmi, S. N. Singh, and S. C. Srivastava, "A synchrophasor assisted frequency and voltage stability based load shedding scheme for self-healing of power system," IEEE Trans. Smart Grid, vol. 2, no. 2, pp. 221-230, Jun. 2011.
[14] L. Karen and N. D. R. Sarma, "Self-healing reconfiguration for restoration of naval shipboard power systems," IEEE Trans. Power Syst., vol. 19, no. 2, pp. 754-762, May 2004.

[15] V. C. Gungor and G. P. Hancke, "Industrial wireless sensor networks: Challenges, design principles, technical approaches," IEEE Trans. Ind. Electron., vol. 56, no. 10, pp. 4258-4265, Oct. 2009.

[16] X. C. Xie, S. Yin, H. J. Gao, and O. Kaynak, "Asymptotic stability and stabilisation of uncertain delta operator systems with timevarying delays," IET Control Theory Appl., vol. 7, no. 8, pp. 1071-1078, May 2013.

[17] H. L. Dong, Z. D. Wang, and H. J. Gao, "Distributed H filtering for a class of Markovian jump nonlinear time-delay systems over lossy sensor networks," IEEE Trans. Ind. Electron., vol. 60, no. 10, pp. 4665-4672, Oct. 2013.

[18] Q. Shafiee et al., "Robust networked control scheme for distributed secondary control of islanded microgrids," IEEE Trans. Ind. Electron., vol. 61 , no. 10, pp. 5363-5374, Oct. 2014.

[19] F. Z. Liu et al., "Networked multirate output feedback control for setpoints compensation and its application to rougher flotation process," IEEE Trans. Ind. Electron., vol. 61, no. 1, pp. 460-468, Jan. 2014.

[20] T. Y. Chai, F. H. Wu, J. L. Ding, and Y. S. Chun, "Intelligent worksituation fault diagnosis and fault-tolerant system for the shaft-furnace roasting process," Proc. Inst. Mech. Eng. I, J. Syst. Control Eng., vol. 221, no. I6, pp. 843-855, Sep. 2007.

[21] M. N. Nounou and B. R. Bakshi, "On-line multiscale filtering of random and gross errors without process models," AIChE J., vol. 45, no. 5, pp. 1041-1058, May 1999.

[22] W. P. Wagner, J. Ottto, and Q. B. Chung, "Knowledge acquisition for expert systems in accounting and financial problem domains," Knowl.-Based Syst., vol. 15, no. 8, pp. 439-447, Nov. 2002

[23] W. Li and Y. Hori, "An algorithm for extracting fuzzy rules based on RBF neural network," IEEE Trans. Ind. Electron., vol. 55, no. 4, pp. 1269 1276, Jun. 2006.

[24] Y. F. Wang, D. H. Wang, and T. Y. Chai, "Extraction and adaptation of fuzzy rules for friction modeling and control compensation," IEEE Trans. Fuzzy Syst., vol. 19, no. 4, pp. 682-693, Aug. 2011.

[25] K. J. Astrom, J. J. Anton, and K. E. Arzen, "Expert control," Automatica, vol. 22, no. 3, pp. 277-286, Mar. 1986.

[26] D. Soumitra, B. Wierenga, and A. Dalebout, "Case-based reasoning systems: From automation to decision-aiding and stimulation," IEEE Trans. Knowl. Data Eng., vol. 9, no. 6, pp. 911-922, Nov./Dec. 1997.

[27] B. Baruque, E. Corchado, A. Mata, and J. M. Corchado, "A forecasting solution to the oil spill problem based on a hybrid intelligent system," Inf. Sci., vol. 180, no. 10, pp. 2029-2043, May 2010.

[28] C. H. Liu and H. C. Chen, "A novel CBR system for numeric prediction," Inf. Sci., vol. 185, no. 1, pp. 178-190, Feb. 2012.

[29] A. Bahga and V. K. Madisetti, "Analyzing massive machine maintenance data in a computing cloud," IEEE Trans. Parallel Distrib. Syst., vol. 23, no. 10, pp. 1831-1843, Oct. 2012.

[30] M. H. Tan and T. Y. Chai, "Modelling of the laminar cooling process with case-based reasoning," Control Theory Appl., vol. 22, no. 2, pp. 248-253, Feb. 2005.

[31] F. Fernandez-Riverola, F. Diaz, and J. M. Corchado, "Reducing the memory size of a fuzzy case-based reasoning system applying rough set techniques," IEEE Trans. Syst., Man, Cybern., vol. 37, no. 1, pp. 138-146, Jan. 2007.

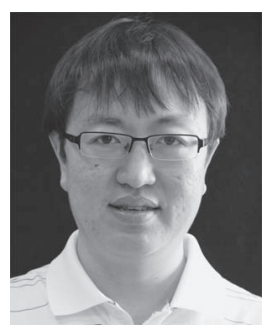

Zhiwei Wu received the B.S. degree in electronic and information engineering from Dalian Nationalities University, Dalian, China, in 2004, and the M.S. degree in control theory and engineering from Shenyang University of Chemical Technology, Shenyang, China, in 2007. $\mathrm{He}$ is currently working toward the Ph.D. degree in control theory and engineering at the State Key Laboratory of Synthetical Automation for Process Industries, Northeastern University, Shenyang, China.

His current research interests include operational control for complex industry processes and industrial embedded control systems. 


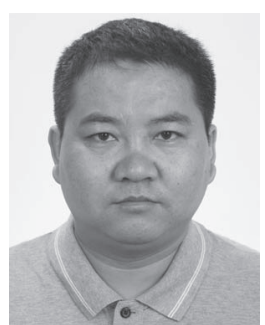

Yongjian Wu received the B.S. and M.S. degrees from Shenyang University of Chemical Technology, Shenyang, China, in 2000 and 2003, respectively, and the Ph.D. degrees from Northeastern University, Shenyang, in 2012.

$\mathrm{He}$ is currently a Lecturer with the State Key Laboratory of Synthetical Automation for Process Industries, Northeastern University, China. His research interests include intelligent control and modeling for complex industry processes.

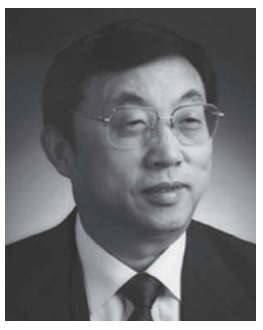

Tianyou Chai (M'90-SM'97-F'08) received the Ph.D. degree in control theory and engineering from Northeastern University, Shenyang, China, in 1985.

In 1988, he became a Professor with Northeastern University. $\mathrm{He}$ is the Founder and Director of the Center of Automation, which became a National Engineering and Technology Research Center and a State Key Laboratory. $\mathrm{He}$ has published 144 peer-reviewed international journal papers. He has developed control technologies with applications to various industrial processes. His current research interests include modeling, control, optimization, and integrated automation of complex industrial processes.

Dr. Chai is a member of the Chinese Academy of Engineering, an IFAC Fellow, and Director of the Department of Information Science of the National Natural Science Foundation of China. For his contributions, he has won four prestigious awards of the National Science and Technology Progress and National Technological Innovation and the 2007 Industry Award for Excellence in Transitional Control Research from the IEEE Multiple-conference on Systems and Control.

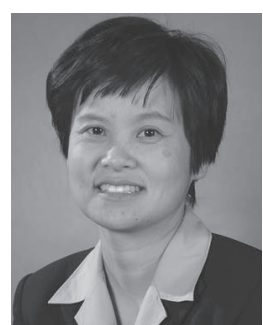

Jing Sun (F'04) received the B.S. and M.S. degrees from the University of Science and Technology of China, Hefei, China, in 1982 and 1984, respectively, and the Ph.D. degree from the University of Southern California, Los Angeles, CA, USA, in 1989.

From 1989 to 1993, she was an Assistant Professor with the Department of Electrical and Computer Engineering, Wayne State University, Detroit, MI, USA. In 1993, she joined the Ford Research Laboratory, where she worked in the Powertrain Control Systems Department. After spending almost ten years in industry, she returned to academia and joined the faculty of the College of Engineering at the University of Michigan, Ann Arbor, MI, in 2003, where she is currently a Professor with the Department of Naval Architecture and Marine Engineering and the Department of Electrical Engineering and Computer Science. Her research interests include system and control theory and its applications to marine and automotive propulsion systems. She holds 37 U.S. patents and has coauthored a textbook on robust adaptive control.

Dr. Sun is one of the three recipients of the 2003 IEEE Control Systems Technology Award. 